\title{
Los servicios personalizados de información de actualidad: estudio de usuarios del periódico Avui.cat
}

\author{
Por Amparo Frías-Castillo y Carina Rey-Martín
}

Resumen: Los estudios de usuarios de los medios de comunicación en la Red, permiten preguntarles de manera directa diferentes cuestiones, en este caso, sobre los servicios personalizados de noticias de actualidad. La encuesta de satisfacción realizada a los lectores del diario Avui.cat ha puesto de manifiesto cómo los servicios personalizados potencian e incrementan el interés por la información y una mayor dedicación a la lectura de noticias, la adquisición del periódico en papel, o la suscripción a otros servicios de noticias.

Palabras clave: Servicios personalizados, Satisfacción, Internet, Información de actualidad, Medios de comunicación, Diarios digitales, Estudios de usuarios, Avui.cat.

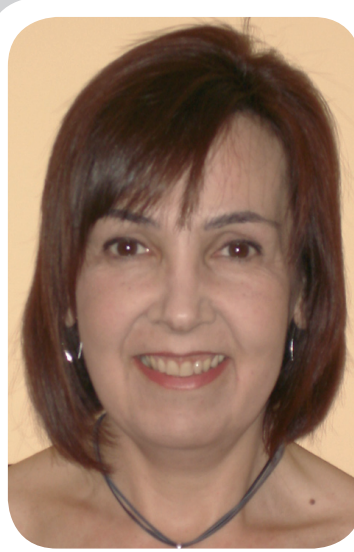

Amparo Frías-Castillo es doctora por la Universitat de Barcelona y licenciada en documentación por la Universitat Oberta de Catalunya. Ha participado en el proyecto de investigación sobre la productividad científica de género de la Facultad de Biblioteconomía y Documentación de la Universitat de Barcelona, es autora de artículos sobre la biblioteca virtual, los servicios personalizados de información de actualidad y las cartas de servicio, y es miembro del grupo de investigación Denea (Detección de Necesidades de la Audiencia) de la UB.

Title: Personalised news services: user study of Avui.cat newspaper readers

Abstract: User studies permit online media to ask users various questions directly, in this case about the benefits to them of personalised news services. The satisfaction survey of Avui.cat readers has revealed how personalised services enhance and increase interest in news information and dedication to reading news, buying the newspaper, or subscribing to other news services.

Keywords: Personalised services, Satisfaction, Net, News information, Mass media, Digital newspapers, User studies, Avui.cat.

Amparo Frías-Castillo; Carina Rey-Martín. "Los servi-

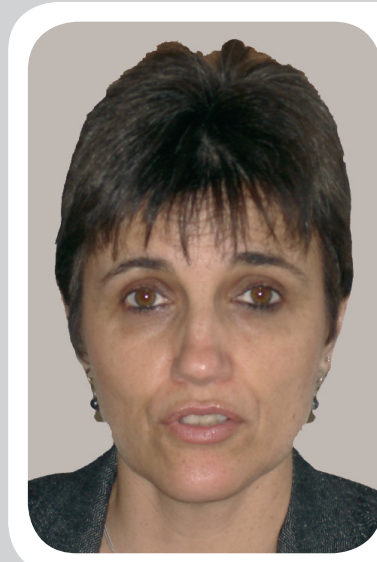

Carina Rey-Martín es doctora por la Universidad Complutense de Madrid y profesora titular de la Facultad de Biblioteconomía y Documentación de la Universitat de Barcelona. Ha participado en varios proyectos de investigación y es autora de diversas publicaciones en diferentes líneas de investigación, como la planificación y gestión de la biblioteca universitaria, la calidad y el marketing en los servicios bibliotecarios. Ha dirigido varias tesis doctorales y es miembro del grupo de investigación Denea (Detección de Necesidades de la Audiencia) de la UB.

cios personalizados de información de actualidad: estudio de usuarios del periódico Avui.cat”. En: El profesional de la información, 2008, julio-agosto, v. 17, n. 4, pp. 437-442.

DOI: 10.3145/epi.2008.jul.11

\section{Introducción}

LA PERSONALIZACIÓN DE LOS CONTENIDOS INFORMATIVOS es una fórmula que los medios de comunicación en la Red están utilizando para conseguir la satisfacción y fidelización de los usuarios. Uno de los productos generados por los medios para poner a disposición de los lectores las noticias de su interés son los servicios personalizados, entendidos como mensajes de texto y/o imágenes enviados a través de los dispositivos móviles de agenda electrónica, teléfono móvil y correo electrónico, principalmente.
Uno de los objetivos de la personalización, según Alfons Cornella, es ofrecer una respuesta específica y concreta a las necesidades de un usuario mediante un servicio interactivo estableciendo un diálogo con el cliente. La materialización de éste diálogo, como afirma el citado autor, puede llevarse a cabo a través de la elección o la definición. La opción de escoger siempre vendrá dada por la existencia de una lista de posibilidades limitada que permita una selección. En cambio, la definición del servicio significa que será el usuario quien expondrá sus necesidades o deseos y en función de éstos se creará una personalización específica para el mismo.

\subsection{Antecedentes}

Los estudios que abordan análisis sobre diferentes aspectos de los diarios digitales son numerosos en nuestro país; por ejemplo, Armañanzas, Armentia, Cabrera, Canga, Díaz-Noci, Guallar, Jiménez, López-Carreño, Meso, Salaverría, etc. Más concretamente, los servicios de información de actualidad han sido estudiados por diversos autores y bajo distintas denominaciones, como la de servicios de valor añadido (SVA). Àn- 
gels Jiménez los define como los que proporciona la prensa digital y que la impresa no puede ofrecer al lector por las limitaciones del soporte documental. Rosana LópezCarreño manifiesta que los SVA personalizados son aquellos que posibilitan establecer perfiles de usuario para obtener una difusión selectiva de información periodística. Manuela Caridad-Valdés incluye el aspecto de la correspondencia del SVA con las necesidades informativas de los usuarios.

Por otro lado, los estudios de usuarios han sido definidos por Elías Sanz, entre otros, como: "los que tratan de analizar cualitativa y cuantitativamente los hábitos de información de los usuarios, mediante la aplicación de distintos métodos, entre ellos los matemáticos -principalmente estadísticos-a su consumo de información". La definición incorpora el término hábitos de información, muy adecuado para los lectores de prensa ya que se trata de una costumbre adquirida diariamente. No obstante resulta necesario preguntar a los usuarios sobre la satisfacción de sus necesidades informativas, ya que las noticias generadas por la propia actualidad pueden hacer variar su interés.

Autores como Aurora González-Teruel entre otros abogan por los estudios de usuarios para facilitar la planificación y mejora de los sistemas de información; y Andy Exon los enfoca hacia la conducta de los usuarios y no usuarios de la información, de los sistemas y también de los servicios.

\section{“El diálogo con el usuario permite detectar qué desea o necesita para sentirse satisfecho informativamente, desde un punto de vista profesional y personal"}

Los medios de comunicación en la Red realizan estudios de usuarios con una cierta periodicidad, pero en general la publicación de los resultados se circunscribe al propio medio, y en pocas ocasiones se 11eva a cabo una divulgación de los mismos.

\subsection{Servicios personalizados de información}

El estudio de los servicios personalizados ofrecidos por los medios de comunicación españoles en la Red nos ha permitido establecer cuatro modelos, en función de las características del nivel de interacción que el medio permite al usuario para la selección de noticias de actualidad de su interés (FríasCastillo):

- Recepción de titulares: la selección de los contenidos de actualidad se realiza por parte del medio, sin la posibilidad de elegir los temas de interés del usuario, ya que se trata de un servicio estándar.

- Selección de secciones o boletines: el usuario suscrito puede escoger entre diversas secciones informativas, a partir de una tabla predeterminada por el medio. En otros casos, el suscriptor tiene la opción de elegir entre diferentes boletines temáticos que hacen referencia a una determinada sección.

- Palabras clave: la recepción de noticias se realiza a partir de los términos de búsqueda introducidos en el formulario de suscripción por parte del lector y posicionados en cualquier lugar del texto (título, entradilla o texto de la noticia).

- Espacio de información personal: permite la adaptación de la pantalla de presentación del servicio, para la visualización de la información elegida por el usuario y con el orden que éste decida. Las noticias se presentan clasificadas en secciones temáticas, que el lector selecciona en base a sus intereses y/o necesidades informativas.
Asimismo, en la citada investigación se estudiaron los sistemas de recepción de estos servicios, mediante las tecnologías push (automática) y pull (inducida) utilizadas por los medios.

\subsection{Avui.cat}

Avui es un periódico fundado en 1976 escrito en lengua catalana y propiedad en estos momentos de la Corporació Catalana de Comunicació SL. Por su parte, la edición digital apareció en 1995, teniendo el honor de ser la primera edición de un medio de información general español en internet.

El servicio personalizado de Avui.cat ofrece al lector la posibilidad de recibir diariamente en su buzón de correo electrónico el Butlletí entre las 5:00 y las 6:00 de la mañana. Este servicio responde a los contenidos periodísticos tradicionales de la prensa general, en el que el suscriptor puede escoger aquellos que respondan a su interés informativo.

Según la tipología de servicios personalizados presentada antes, Avui.cat se enmarca en el modelo de selección de secciones. En cuanto a sistema de recepción utiliza la tecnología automática, ya que se trata de una descarga sistemática de información por el proveedor del servicio. El servidor envía la información al usuario conectado a internet sin que éste la haya solicitado.

\section{Objetivos y metodología}

El 23 de abril de 2007 el periódico Avui.cat presentó una nueva imagen y distribución de contenidos informativos. Los objetivos de nuestro estudio eran conocer el impacto informativo de esa nueva distribución y la satisfacción en referencia a las expectativas depositadas en el periódico y a los servicios ofrecidos. El análisis cuantitativo y cualitativo ha permitido obtener los datos que se presentan más adelante. 
Pasados casi cinco meses desde la incorporación del nuevo diseño, el pasado 16 de octubre se incluyó en la web del medio una pregunta para que los lectores pulsaran sobre ella y entrasen a responder la encuesta de satisfacción.

La técnica utilizada para la recogida de datos ha sido el cuestionario estructurado, un listado de preguntas preestablecidas para formular a los encuestados a través de la Red. Esta técnica se considera el soporte más adecuado para medir las opiniones, actitudes, intenciones, valores, juicios, sentimientos, expectativas, etc. El cuestionario es un instrumento de investigación que mediante procedimientos estandarizados de interrogación, permite la comparación de respuestas y obtener variadas medidas cuantitativas de aspectos objetivos y subjetivos de una población, tal y como afirma Ruiz Abellán. Cabe mencionar que el cuestionario fue redactado en catalán, idioma del periódico, y que se presenta aquí traducido al castellano.

La encuesta se estructuró en tres bloques: intereses informativos generales (12 preguntas); información sobre el servicio de Butlletí con 3 preguntas sólo para suscriptores; y satisfacción en referencia a la web (5 preguntas). Los bloques tenían la finalidad de facilitar la respuesta a los usuarios, ya que el contenido de cada uno de ellos hacía referencia a aspectos concretos agrupados temáticamente. Se incluyeron mayoritariamente preguntas cerradas en las que el usuario escogía una respuesta de un conjunto preestablecido, con una opción de respuesta abierta en algunos casos.

La invitación a la respuesta de la encuesta por parte del periódico se manifestaba en forma de pregunta: ¿Quieres ayudarnos a mejorar Avui.cat?

La pulsación del enlace abría una nueva ventana en la que aparecía la batería de preguntas. La configuración informática de la en- cuesta no permitía dejar respuestas en blanco, por lo tanto, se considera que algunas de éstas no habrán sido fruto de la reflexión de las preguntas, sino de la simple necesidad de pasar a una nueva página, con la finalidad de terminar la encuesta.

En este artículo se incluyen las preguntas de la encuesta que hacen referencia al servicio Butlletí (para sus suscriptores), ya que se trata del servicio personalizado de recepción de noticias que el periódico tiene a disposición de sus usuarios y que requiere de su registro, así como de la disposición de una dirección de correo electrónico para recibir todas o alguna de las 8 secciones escogidas:

$$
\begin{aligned}
& \text { - Opinión } \\
& \text { - Economía } \\
& \text { - Mundo y política } \\
& \text { - Cultura y comunicación } \\
& \text { - Sociedad y tecnología } \\
& \text { - Deportes } \\
& \text { - Más cerca, y } \\
& \text { - Gente. }
\end{aligned}
$$

De la misma forma, permite seleccionar los días de la semana que el usuario desea recibirlos. En el momento de realizar la encuesta, se contaba con 8.115 suscriptores, de los que un $1,5 \%$ (122) ha contestado el cuestionario.

Asimismo se incorporan las respuestas correspondientes a la satisfacción obtenida respecto del contenido y las necesidades informativas, del total de lectores de la web, que durante los 21 días en que se mantuvo activa la encuesta se contabilizó una media de 18.241 usuarios únicos (datos obtenidos de la OJD, Oficina de Justificación de la Difusión), de los que un $1,66 \%$ (302) respondieron la encuesta.

\section{http://www.ojd.es}

Según diversos estudios, la respuesta de encuestas a través de la Red se estima entre el 8 y el $10 \%$ de la población consultada. En este caso, el porcentaje alcanzado ha sido inferior al mencionado, no obstante, se considera aceptable. El número de respuestas obtenidas obliga a realizar una extrapolación con un menor número de datos reales, pero igualmente interesantes para iniciar líneas de mejora, a partir de las sugerencias y respuestas obtenidas.

\section{Resultados}

\subsection{Bloque información sobre el servicio Butlletí}

Se transcriben a continuación las preguntas y resultados obtenidos y referidos al objeto de estudio.

1. ¿Qué contenidos desearía recibir por medio del Butlletí?

El $40 \%$ de los suscriptores desearía recibir los titulares del Avui en papel, el $21 \%$ el nombre del autor de los artículos; el 14\% las propuestas de la agenda del día y también la encuesta del día; el $8 \%$ el horóscopo y 'otros' el 3\%.

Las respuestas manifiestan un interés por la inclusión de contenidos informativos que en este momento no forman parte del servicio y demuestran la heterogeneidad de los lectores del periódico.

2. ¿En qué grado ha influido la recepción del Butlletí en el interés por las noticias de actualidad?

La respuesta se presenta mediante una valoración numérica tipo Likert o escala de medida de actitudes de variables cualitativas (el 1 representa una respuesta negativa y el 7 una positiva).

El resultado ha sido altamente favorable, ya que el $40 \%$ de los suscriptores responden con una puntuación entre el cinco y el siete, o valoración alta (figura 1), el 20\% ha puntuado con un cuatro, el $9 \%$ con un tres, el $7 \%$ con un dos y un $24 \%$ con un uno. Éste último dato invita a redefinir el servicio para obtener un mayor grado de satisfacción en posteriores consultas. 


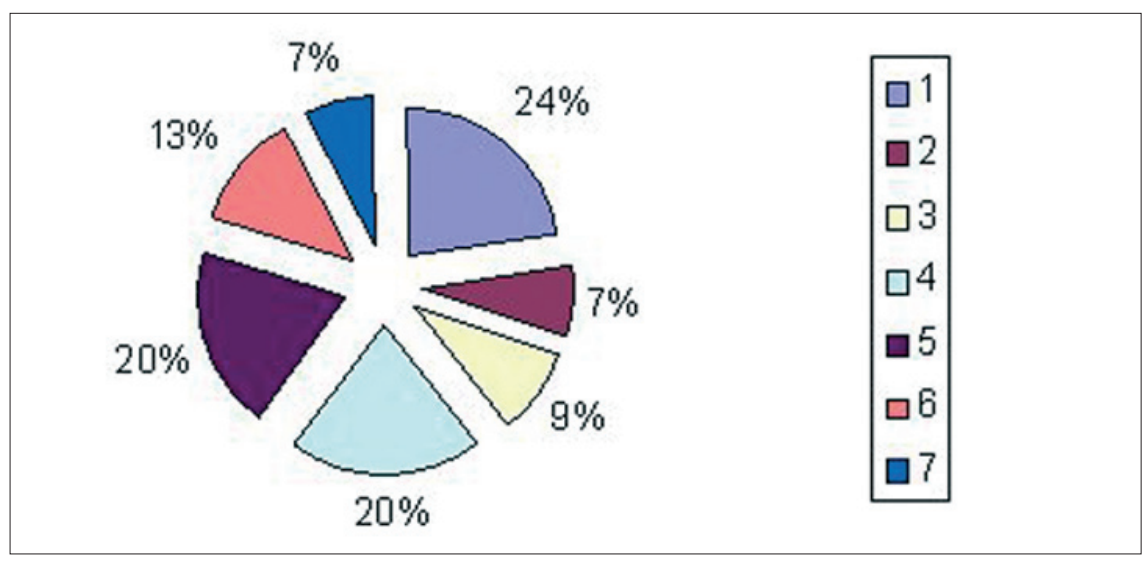

Figura 1

El perfil de los usuarios obtenido a partir de la combinación de la edad y el interés manifestado por las noticias presenta las valoraciones más altas centradas en los lectores nacidos en los 70 y 80 (población de entre 25 y 35 años). Asimismo, en este período se concentran las notas más negativas, donde destaca un alto número de suscriptores que consideran que el Butlletí no ha servido para incrementar su interés por las noticias de actualidad. Las valoraciones de los nacidos en los 60 (45 años) son un tanto conservadoras, ya que presentan uniformidad en las puntuaciones.

3. ¿Cómo se ha manifestado este interés?

La respuesta permite tres opciones entre las que destaca el 65\% de los suscriptores que manifiestan dedicar un mayor tiempo de lectura a las noticias; el $29 \%$ han pasado a ser compradores del diario en papel y el $6 \%$ ha realizado la suscripción a otros servicios de noticias (figura 2).

Los resultados obtenidos a partir de ésta pregunta pueden considerarse altamente positivos, ya que muestran que un servicio de noticias en el que se permite al usuario seleccionar el contenido informativo favorece e incrementa su interés por las noticias de actualidad.

El análisis de la edad de los suscriptores del Butlletí y la manifestación del interés por las noticias, señala los nacidos en los 60, 70 y 80 (de 45 a 25 años) como los que dedican más tiempo a la lectura de

\section{"Las personas nacidas en los 60, 70 y 80 manifiestan ser las que dedican más tiempo a la lectura de \\ noticias"}

noticias; mientras que en lo referente a la adquisición del periódico en papel destacan los subscriptores de los 70 y 80, población comprendida entre los 25 y 35 años.

A partir de estos datos se puede concluir que la recepción de noticias de interés para el suscriptor provoca un efecto positivo y le incentiva a la lectura, y por lo tanto al consumo de información nueva o más desarrollada.

\subsection{Bloque satisfacción en refe- rencia a la web}

Se han escogido dos cuestiones referidas a la satisfacción de la web, que analizan las necesidades informativas y diferentes aspectos generales, con el fin de obtener una imagen descriptiva del cumplimiento de las expectativas de los lectores.

1. ¿En comparación con sus expectativas, en qué medida el sitio web ha satisfecho sus necesidades de información?

El 74\% de los usuarios han manifestado que han sido resueltas y un $16 \%$ que las han excedido positivamente (figura 3). Un 10\% han contestado que el nuevo sitio web ha resultado decepcionante, y manifiestan de forma mayoritaria la necesidad de encontrar noticias más específicas y desarrolladas; la no actualización continua de los contenidos de la web; la inclusión de más artículos de opinión, de información local y política; las dificultades para encontrar la información a través del buscador; y la falta de rigor lingüístico en los contenidos informativos. 


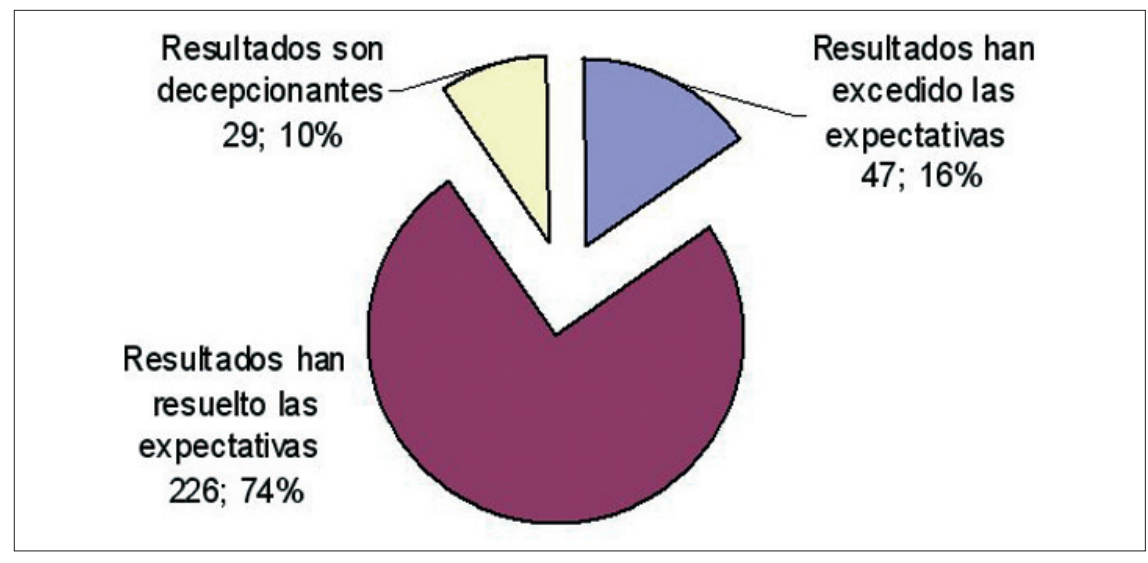

Figura 3

\section{"La recepción de noticias de interés para el suscriptor provoca un efecto positivo y le incentiva a la lectura"}

Ese $10 \%$ de usuarios que consideran no satisfechas sus necesidades corresponde a diversas décadas de nacimiento. El $16 \%$ pertenece a los 50, el $26 \%$ a los 60 , el $23 \%$ a los 70 y el $20 \%$ a los 80 . Se considera que la insatisfacción se reparte equitativamente en diversos grupos de edad, y si bien afecta a un pequeño número de lectores, se debe tener en cuenta el amplio abanico de edades.

\section{2. ¿Cuál es su nivel de satisfac-} ción en los siguientes aspectos del sitio web?

En este caso se escogió también la respuesta de tipo Likert (con valores entre uno y siete). Respecto del nivel de satisfacción general de los usuarios del servicio Butlletí, y concretamente en referencia a la gestión y al grado de personalización, se debe mencionar que los valores 5-7, considerados en el tramo alto de la tabla, se sitúan por debajo del $20 \%$ de los subscriptores del servicio.

Este dato induce a pensar en la necesidad de facilitar la visibilidad del servicio para hacerlo más accesible, así como la gestión del mismo y potenciar el grado de personalización con nuevos aspectos que denoten una singularidad del servicio y conseguir unas valoraciones más favorables en próximas consultas.

\section{Conclusiones}

La realización de un estudio a los lectores del periódico Avui.cat ha permitido conocer, desde la opinión de los propios usuarios del diario, cuál es su grado de satisfacción en varios aspectos consultados.

Uno de los datos más relevantes de la encuesta es el bloque sobre el servicio personalizado de noticias Butlletí, que ha puesto de manifiesto un incremento del interés por las noticias de actualidad y una mayor dedicación de tiempo a su lectura en un $65 \%$ de los casos; además se ha detectado que un $29 \%$ de lectores han pasado a adquirir el periódico en papel, y un $6 \%$ se ha suscrito a otros servicios de noticias.

El estudio ha revelado la necesidad de poner al alcance de los lectores servicios personalizados de noticias que faciliten la selección de contenidos para satisfacer sus inquietudes informativas. Estos servicios incrementan la predisposición del lector para una mayor dedicación a la lectura, y quizá otros diarios digitales deberían plantearse también su inclusión.
Por último, cabe señalar que la realización de estudios de usuarios permite detectar a través del diálogo con ellos, qué desean o necesitan para sentirse satisfechos informativamente, tanto desde un punto de vista profesional como personal. Este instrumento ha de considerarse como una herramienta válida y directa que posibilita el conocimiento de los intereses de los usuarios, y -como se ha demostrado en el caso expuesto- resulta muy positivo para persuadir a la lectura.

\section{Bibliografía}

Alvira, Francisco. La encuesta: una perspectiva general metodológica. Madrid: CIS, 2004, ISBN 84-7476-370-3.

Armañanzas, Emy; Díaz-Noci, Javier; Meso, Koldo. El periodismo electrónico: información y servicios multimedia en la era del ciberespacio. Barcelona: Ariel, 1996, ISBN 84-344-1270-5.

Armentia-Vizuete, José Ignacio, et al. El diario digital: análisis de los contenidos textuales, aspectos formales y publicitarios. Barcelona: Bosch, 2000, ISBN 84-7676-738-2.

Cabrera-González, Ma Ángeles. La prensa online. Barcelona: CIMS, 2000, ISBN 84-8411540-2.

Calva, Juan José. Las necesidades de información: fundamentos teóricos y métodos. México: UNAM, 2004, ISBN 970-32-1798-2.

Canga, Jesús; Coca, César. Diarios digitales: apuntes sobre un nuevo medio. Bilbao: Universidad del País Vasco. Servicio editorial, 2000, ISBN 84-8373-211-4.

Cornella, Alfons. Infonomia.com: la empresa es información. Bilbao: Deusto, 2000, ISBN $84-$ 234-1760-3.

Díaz-Noci, Javier; Meso-Ayerdi, Koldo. Medios de comunicación en internet: guía de navegación. Madrid: Anaya Multimedia, 1997, ISBN 84-415-0140-8.

Díaz-Noci, Javier; Meso-Ayerdi, Koldo. Periodismo en internet: modelos de la prensa digital. Bilbao: Universidad del País Vasco. Servicio Editorial, 1999, ISBN [84-8373-161-4].

Díaz de Rada, Vidal. Manual de trabajo de campo en la encuesta. Madrid: CIS, 2005, ISBN 84-7476-388-6.

Dillman, Don A. Mail and internet surveys: the tailored design method. New York: Wiley \& Sons, 2000, ISBN 0-471-32354-3.

Exon, Andy. Getting to know the user better. En: Aslib proceedings, 1978, v. 30, n. 10, pp. 352-364.

Frías-Castillo, Amparo. Els estudis d'usuaris en el serveis personalitzats als mitjans de comunicació a internet. Consultado en: 27-01-08. http://www.tesisenxarxa.net/TESIS_UB/AVAILABLE/TDX-1205107-133615//03.AFC_ CAP\%CDTOL_3.pdf 
Frías-Castillo, Amparo; Rey-Martín, Carina Los servicios personalizados de información de actualidad en los medios de comunicación españoles a través de internet. Consultado en: 18-12-07.

http://eprints.rclis.org/archive/00012245/

González Teruel, Aurora. Los estudios de necesidades y usos de la información: fundamentos y perspectivas actuales. Gijón: Trea, 2005.

Guallar, Javier. "Prensa digital en 2006". En: Апиа rio ThinkEPI, 2007. Consultado en: 15-04-08. http://eprints.rclis.org/archive/00008388/

Hayes, Bob E. Cómo medir la satisfacción del cliente: desarrollo y utilización de cuestionarios. Barcelona: Gestión 2000, 2002, ISBN 84-8088696-X.

Jiménez, Àngels. Estudi de la gestió documental de la informació en els serveis de valor afegit dels mitjans de comunicació a internet: el cas de la premsa diària a l'estat espanyol. Consultado en: 15-04-08.

http://www.tdcat.cesca.es/

Jiménez, Àngels; González, Alfons. "Gestió documental de la informació en els serveis de valor afegit de la premsa espanyola a internet'. En: 7es. Jornades catalanes de documentació Les biblioteques i els centres de documentació al segle XXI: peça clau de la societat de la informació. Consultado en: 15-04-08.
http://www.cobdc.org/jornades/7JCD/home-cat. htm

López-Carreño, Rosana. "Análisis taxonómico de los portales periodísticos españoles". En: Anales de documentación, 2004, v. 7, pp. 123 140

Martín, Carmen; Sanz, Elías. "Aplicación de dos técnicas de recogida de datos para la realización de estudios de usuarios". En: Investigación bibliotecológica, 2001, v. 15, n. 30, pp. 194-213.

Rey, Carina. "La satisfacción del usuario: un concepto en alza". En: Anales de documentación, 2000, v. 3, pp. 139-153.

Rojas, Antonio J.; Fernández, Juan-Sebastián. Investigar mediante encuestas: fundamentos teóricos y aspectos prácticos. Madrid: Síntesis, 1998, ISBN 84-7738-598-X

Ruiz, Joaquín; Izquierdo, Mónica. "El cuestionario estructurado como herramienta básica para la evaluación de las instituciones documentales". En: Los sistemas de información al servicio de la sociedad. Valencia: Fesabid, 1998, pp. 779-789.

Salant, Priscilla. How to conduct your own survey. New York: Wiley, 1994, ISBN 0-471-01273-

Salaverría, Ramón (coord.). Cibermedios. El impacto de internet en los medios de comuni- cación en España. Sevilla: Comunicación Social ediciones y publicaciones, 2005.

Sánchez, Juan-Javier. La bondad de la encuesta: el caso de la no respuesta. Madrid: Alianza Editorial, 2000, ISBN 84-206-5764-6.

Sanz, Elías. Manual de estudios de usuarios. Madrid: Fundación Germán Sánchez Ruipérez, 1994, ISBN 84-86168-93-7.

Sanz, Elías. "La realización de estudios de usuarios: una necesidad urgente". En: Revista general de información y documentación, 1993, v. 3, n. 1. Consultado en: 18-12-07.

http://www.ucm.es/BUCM/revistas/byd/ 11321873/articulos/RGID9393120155A. PDF

Amparo Frías-Castillo, Facultad de Biblioteconomía y Documentación, Universitat de Barcelona.

frias.amparo@gmail.com

Carina Rey-Martín, Facultad de Biblioteconomía y Documentación, Universitat de Barcelona. carina.rey@ub.edu

\section{¿Sabías que $\mathbf{Z P}$ tiene blog?}

\section{Se llama "Zona de notas"}

\section{http://www.elprofesionaldelainformacion.com/notas/}

En él puedes seguir toda la actualidad

y noticias sobre

"El profesional de la información": novedades,

comentarios sobre los contenidos, call for papers, reseñas de actividades,

noticias sobre EPI en otros medios, etc.

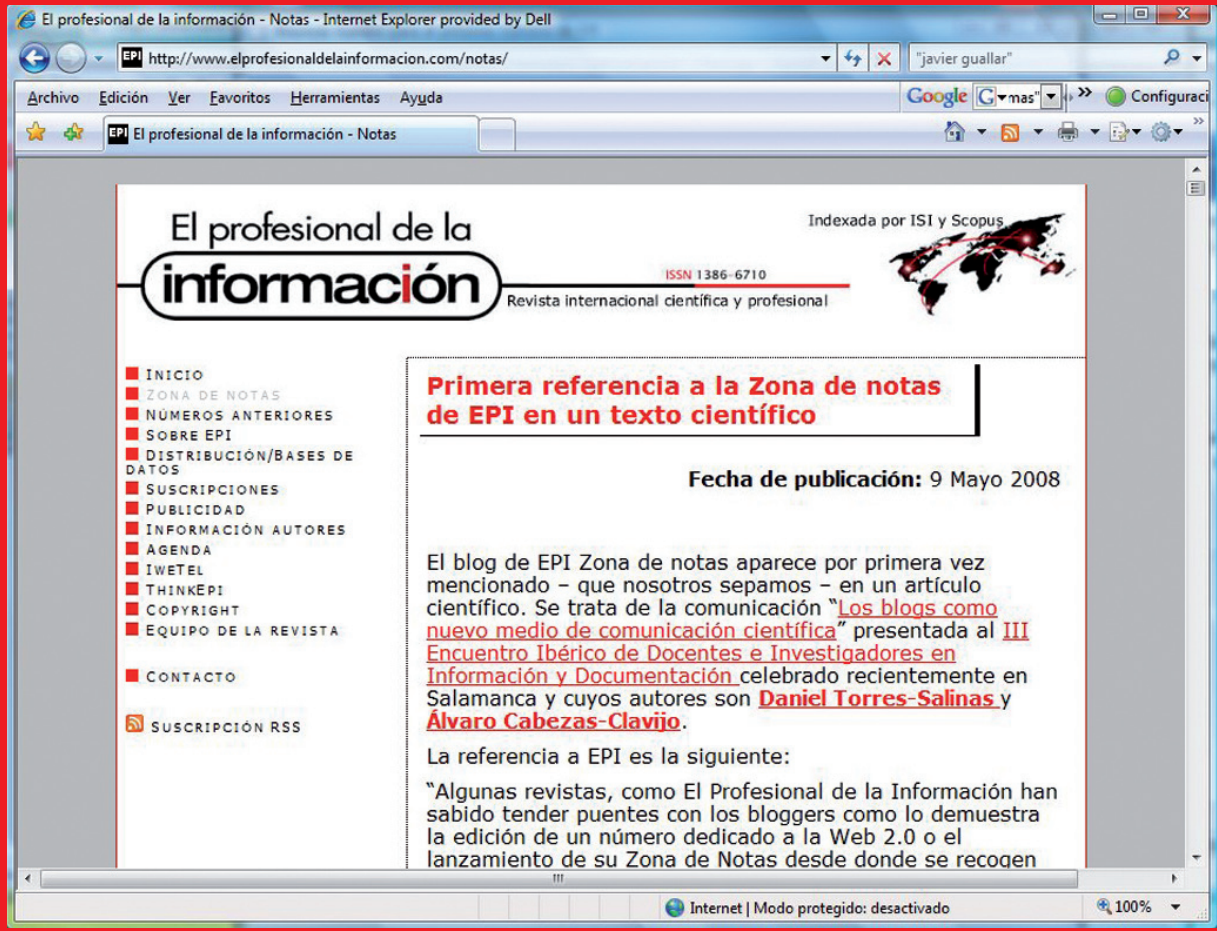

